University of Wollongong

Research Online

Faculty of Informatics - Papers (Archive)

Faculty of Engineering and Information

Sciences

February 2001

\title{
Boundary filters for size-limited paraunitary filter banks with maximum coding gain and ideal DC behavior
}

Alfred Mertins

University of Wollongong, mertins@uow.edu.au

Follow this and additional works at: https://ro.uow.edu.au/infopapers

Part of the Physical Sciences and Mathematics Commons

\section{Recommended Citation}

Mertins, Alfred: Boundary filters for size-limited paraunitary filter banks with maximum coding gain and ideal DC behavior 2001.

https://ro.uow.edu.au/infopapers/25

Research Online is the open access institutional repository for the University of Wollongong. For further information contact the UOW Library: research-pubs@uow.edu.au 


\title{
Boundary filters for size-limited paraunitary filter banks with maximum coding gain and ideal DC behavior
}

\author{
Abstract \\ This paper presents boundary optimization techniques for the processing of arbitrary-length signals with \\ paraunitary multirate filter banks. The boundary filters are designed to maximize the coding gain while \\ providing an ideal DC behavior where all filters except the low-pass filters have zero mean. Moreover, \\ solutions are presented that have similar frequency responses as the original subband filters. The \\ proposed methods give direct solutions to the problem of finding the optimal boundary filters with \\ maximum coding gain and do not require numerical optimization. Thus, they are even applicable to \\ systems with a large number of subbands and/or very long filter impulse responses.
}

\section{Keywords}

boundary filters, filter banks, multirate signal processing, subband coding

\section{Disciplines}

Physical Sciences and Mathematics

\section{Publication Details}

This article was originally published as: Mertins, A, Boundary filters for size-limited paraunitary filter banks with maximum coding gain and ideal DC behavior, IEEE Transactions on Circuits and Systems II: Analog and Digital Signal Processing, February 2001, 48(2), 183-188. Copyright IEEE 2001. 


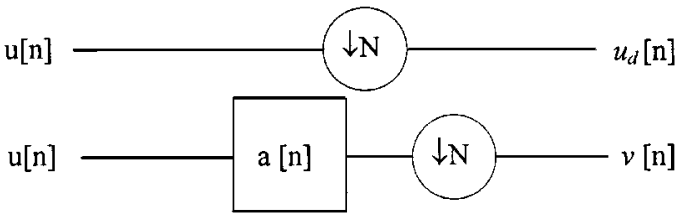

Fig. 4. (a) Decimation. (b) Filtering and decimation.

Substituting (35) into (34), one obtains

$$
\prod_{r=1}^{m} A\left(e^{j\left(\omega / a_{0}^{r}\right)}\right)=\sqrt{a_{0}^{m}}, \quad|\omega| \leq \pi, m \geq 1 .
$$

This relation can be satisfied if the filter $a[n]$ is the ideal low-pass filter having the frequency response defined over the baseband $|\omega| \leq \pi$ by

$$
A\left(e^{j \omega}\right)= \begin{cases}\sqrt{a_{0}} e^{j \theta(\omega)}, & |\omega| \leq \frac{\pi}{a_{0}} \\ 0, & \text { otherwise }\end{cases}
$$

where the phase response $\theta(\omega)$ should satisfy the constraint

$$
\sum_{r=1}^{m} \theta\left(\frac{\omega}{a_{0}^{r}}\right)=2 \pi k, \quad|\omega| \leq \pi, m \geq 1, k \text { integer. }
$$

\section{CONCLUSION}

A filter bank implementation of the discrete time wavelet transform $\operatorname{DTWT}[m, n]$ has been developed. A closed-form expression for the discrete time Fourier transform of the DTWT $[m, n]$ viewed as a sequence in $n$ first was derived. Next, an expression for the frequency-domain outputs of the filter bank structure of Fig. 1 defined by the digital filters $a[n]$ and $b[n]$, was obtained. By equating both expressions, the filter $a[n]$ emerged as an ideal low-pass filter and the filter $b[n]$ as a bandpass filter obtained by time-reversing and complex conjugating the sampled mother wavelet $\psi(n T)$.

\section{REFERENCES}

[1] S. Mallat, A Wavelet Tour of Signal Processing. Boston, MA: Academic, 1998.

[2] G. Strang and T. Nguyen, Wavelets and Filter Banks. Wellesley, MA: Wellesley-Cambridge, 1996.

[3] Y. Meyer, Wavelets: Algorithms and Applications. Philadelphia, PA: SIAM, 1993.

[4] M. Vetterli and J. Kovacevic, Wavelets and Subband Coding. Englewood Cliffs, NJ: Prentice-Hall, 1995.

[5] A. N. Akansu and M. J. Medley, Eds., Wavelet, Subband and Block Transforms in Communication and Multimedia. Boston, MA: Kluwer, 1998.

[6] A. N. Akansu and M. J. T. Smith, Eds., Subband and Wavelet Transforms: Design and Applications. Boston, MA: Kluwer, 1995.

[7] A. N. Akansu and R. A. Haddad, Multiresolution signal decomposition. San Diego, CA: Academic, 1992.

[8] M. J. Shensa, "The discrete wavelet transform: Wedding the 'a trous and Mallat algorithms," IEEE Trans. Signal Processing, vol. 40, pp. 2464-2482, Oct. 1992.

[9] Y. T. Chan, Wavelet Basics. Boston, MA: Kluwer Academic, 1995

[10] A. V. Oppenheim, A. S. Willsky, and I. T. Young, Signals and Systems. Englewood Cliffs, NJ: Prentice-Hall, 1983.

\section{Boundary Filters for Size-Limited Paraunitary Filter Banks with Maximum Coding Gain and Ideal DC Behavior}

\author{
Alfred Mertins
}

\begin{abstract}
This paper presents boundary optimization techniques for the processing of arbitrary-length signals with paraunitary multirate filter banks. The boundary filters are designed to maximize the coding gain while providing an ideal dc behavior where all filters except the low-pass filters have zero mean. Moreover, solutions are presented that have similar frequency responses as the original subband filters. The proposed methods give direct solutions to the problem of finding the optimal boundary filters with maximum coding gain and do not require numerical optimization. Thus, they are even applicable to systems with a large number of subbands and/or very long filter impulse responses.
\end{abstract}

Index Terms-Boundary filters, filter banks, multirate signal processing, subband coding.

\section{INTRODUCTION}

Multirate filter banks are usually designed to process ongoing signals, but it is also of significant interest to use them for the processing of finite-length signals. Applications include segmentation-based audio [1]-[4] and region-based [shape adaptive (SA)] image coding [5], [6]. From a compression point of view, it is desirable to carry out a filter bank analysis of a finite-length signal in a nonexpansive way. This means that the total number of subband samples produced from a timelimited signal should be equal to the number of samples of the signal. Achieving this goal with filter banks, however, requires some additional steps, because the filter impulse responses are overlapping and the transient behavior at the signal boundaries must be taken into account.

Various techniques have been proposed to process finite-length signals, including circular convolution [7], symmetric reflection [3], [8]-[11], and the use of boundary filters [12]-[21]. This paper concentrates on boundary filters and presents novel methods to optimize them with regard to the coding gain. Using boundary filters means that the original filters of the filter bank are replaced by special filters at the boundaries of the signal, which ensure that the entire information on a length- $N$ input signal is contained in a total number of $N$ subband samples. Circular convolution and symmetric reflection can also be interpreted as special forms of boundary filters.

One often aims at designing the filters in a filter bank in such a way that they have a large number of vanishing moments because this ensures good energy compaction properties for low-order polynomial signals and other low-frequency signals. A minimum requirement is usually that all filters except the low-pass have zero mean. Otherwise, a large dc component of the input signal could leak into several bands and could cause problems with the bit allocation in these bands. DC leakage is particularly problematic in image compression where it causes highly visible artifacts. When applying a filter bank to a finite-length signal by using boundary filters, the problem that the boundary filters will usually not satisfy any moment conditions occurs, even if the original filters do. For biorthogonal filter banks, this problem was addressed in [19] and [21], and solutions were proposed that allow one to design boundary filters that match the moments of the original filters

Manuscript received May 2000; revised January 2001. This paper was recommended by Associate Editor B. Baykal.

The author is with the School of Electrical, Computer and Telecommunications Engineering, University of Wollongong, Wollongong, NSW 2522, Australia (e-mail: mertins@uow.edu.au).

Publisher Item Identifier S 1057-7130(01)03050-6. 
up to a certain degree. The work in [21] also states conditions under which both matching moments and paraunitaryness can be obtained and introduces a design method that yields orthogonality and almost matching moments without the need of numerical optimization. The optimality criterion in [21] is related to the one in [15] and [16] and is different from the maximization of the coding gain considered in this paper. In [20], a solution to the paraunitary two-channel case was proposed, which first optimizes the boundary filters and then applies a Householder transform to obtain zero-mean high-pass filters. Due to the Householder step, the final filters are no longer optimal.

This paper shows how boundary filters with maximum coding gain can be designed in a straightforward manner and without the need of numerical optimization. No restrictions on the type of the paraunitary filter bank, the number of channels, and the signal length are imposed. Thus, the proposed methods are applicable to nonlinear phase filter banks and signals of arbitrary length. This is important, as the often used cosine modulated filter banks have nonlinear phase. The design can either be carried out without further constraints or under the constraint of an ideal dc behavior. To control the dc behavior, a projection technique is used. Optimization is then carried out in a second step. It is shown that maximizing the coding gain through optimizing the boundary filters results in an eigenvalue problem, which has a straightforward solution. In addition to maximizing the coding gain, a method is proposed that allows us to find boundary filters that have similar frequency responses as the original subband filters in the filter bank. Note that the coding gain has also been considered in [17] and [22] In [17], numerical optimization was employed to find the boundary filters and no dc constraints were imposed. The use of numerical optimization practically limits the method to systems with a small number of subbands and short filter impulse responses, because the number of unknown parameters rapidly increases with the number of bands and the filter lengths. The proposed method does not suffer from such limitations. In [22], the coding gain was used to optimize the bit allocation for given boundary filters and not to optimize the filters themselves.

This paper is organized as follows. In Section II, the framework for the construction of boundary filters is given and a method to avoid dc leakage is presented. Methods for optimizing the boundary filters are presented in Section III. Section IV presents design examples, and Section $\mathrm{V}$ gives some conclusions.

\section{The CONSTRUCTION OF BOUNDARY FILTERS}

This section first gives a brief outline of size-limited filter banks and the construction of boundary filters via the Gram-Schmidt procedure, followed by a discussion of the degrees of freedom being available for filter optimization when no further restrictions are imposed. Finally, a method to ensure ideal dc behavior is introduced, and the remaining degrees of freedom for further optimization are discussed.

\section{A. Construction of Basic Size-Limited Filter Banks}

We consider an arbitrary signal length $N$ and describe it as

$$
N=K M+s
$$

where $M$ denotes the number of subbands and $K$ and $s$ satisfy $K \in \mathbb{Z}$ and $0 \leq s<M, s \in \mathbb{Z}$. The filter bank analysis of a length- $N$ signal $x(n)$ may then be written as

$$
\boldsymbol{y}=\boldsymbol{H} \boldsymbol{x}
$$

with

$$
\boldsymbol{x}=[x(0), x(1), \ldots, x(N-1)]^{T}
$$

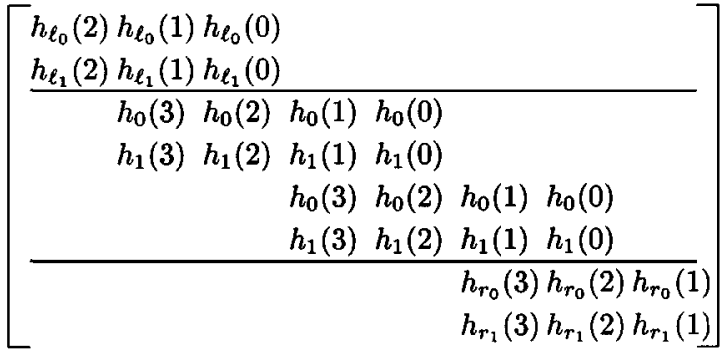

Fig. 1. Example of size-limited analysis matrix $\boldsymbol{H} \cdot M=2, N=8$, length-4 filters.

and

$$
\begin{aligned}
\boldsymbol{y}= & {\left[y_{0}(0), \ldots, y_{M-1}(0), \ldots, y_{0}(K-1), \ldots\right.} \\
& \left.\ldots, y_{M-1}(K-1), y_{0}(K), \ldots, y_{s-1}(K)\right]^{T} .
\end{aligned}
$$

Given the definitions for $\boldsymbol{x}$ and $\boldsymbol{y}$, the $N \times N$ matrix $\boldsymbol{H}$ can be set up to describe the filter bank analysis. Fig. 1 gives an example of $\boldsymbol{H}$ for a two-band decomposition of a length- 8 signal.

The matrix $\boldsymbol{H}$ may be partitioned as

$$
\boldsymbol{H}=\left[\boldsymbol{H}_{1}^{T}\left|\boldsymbol{H}_{2}^{T}\right| \boldsymbol{H}_{3}^{T}\right]^{T}
$$

where the center part contains the original impulse responses of the analysis filters, while the upper and lower parts contain boundary filters. Using this partitioning, the analysis equation (2) can be rewritten as

$$
\boldsymbol{y}_{k}=\boldsymbol{H}_{k} \boldsymbol{x}, \quad k=1,2,3
$$

so that $\boldsymbol{y}=\left[\boldsymbol{y}_{1}^{T}, \boldsymbol{y}_{2}^{T}, \boldsymbol{y}_{3}^{T}\right]^{T}$. Similarly, the synthesis operation can be written as

$$
\hat{\boldsymbol{x}}=\sum_{k=1}^{3} \boldsymbol{G}_{k} \boldsymbol{y}_{k}
$$

where $\boldsymbol{G}_{k}$ are the corresponding partitions of the synthesis matrix $G$, such that $\hat{\boldsymbol{x}}=\boldsymbol{G} \boldsymbol{y}$. Perfect reconstruction (PR) is given if $\boldsymbol{G H}=\boldsymbol{I}$. In particular, if the size-limited filter bank is unitary, we have PR with $\boldsymbol{G}=\boldsymbol{H}^{T}$.

To design a matrix $\boldsymbol{H}$ that satisfies $\boldsymbol{H}^{T} \boldsymbol{H}=\boldsymbol{I}$, the Gram-Schmidt procedure can be used [13], [14]. In this method, in a first step, one sets up an appropriately sized matrix $\boldsymbol{F}$, which describes a filter bank analysis with the given analysis filters. In a second step, this matrix is truncated to size $N \times N$. Finally, the rows of the truncated matrix are orthogonalized with the Gram-Schmidt procedure to yield an orthonormal matrix $\boldsymbol{H}$ satisfying $\boldsymbol{H}^{T} \boldsymbol{H}=\boldsymbol{I}$. Fig. 2 shows an example of $\boldsymbol{F}$ and its truncation. The orthogonalization of the truncated matrix yields the matrix $H$ depicted in Fig. 1. The extension to the $M$-channel case is straightforward. Note that for a paraunitary filter bank, the nontruncated rows of $\boldsymbol{F}$ are mutually orthogonal and orthogonal to the truncated ones.

\section{B. Degrees of Freedom}

We assume that the PR condition $\boldsymbol{G H}=\boldsymbol{H} \boldsymbol{G}=\boldsymbol{I}$ is satisfied with $\boldsymbol{G}=\boldsymbol{H}^{T}$. The submatrices then satisfy $\boldsymbol{H}_{k} \boldsymbol{G}_{k}=\boldsymbol{I}_{k}$, where $\boldsymbol{I}_{k}$ are identity matrices of appropriate size. Terms of the form $\boldsymbol{G}_{k} \boldsymbol{H}_{k}$ describe orthogonal projections onto the column spaces of $\boldsymbol{G}_{k}$. During optimization, the aim is to replace the matrices $\boldsymbol{H}_{1}$ and $\boldsymbol{H}_{3}$ by new, better matrices $\tilde{\boldsymbol{H}}_{1}$ and $\tilde{\boldsymbol{H}}_{3}$. Clearly, we then also have to replace the corresponding synthesis partitions $\boldsymbol{G}_{1}$ and $\boldsymbol{G}_{3}$ by new ones and have to ensure that $\tilde{\boldsymbol{H}}_{k} \tilde{\boldsymbol{G}}_{k}=\boldsymbol{I}_{k}$ and $\tilde{\boldsymbol{G}}_{k} \tilde{\boldsymbol{H}}_{k}=\boldsymbol{G}_{k} \boldsymbol{H}_{k}$. This makes clear that both $\boldsymbol{H}_{k}$ and $\tilde{\boldsymbol{H}}_{k}$ must have the same row space and that the rows 


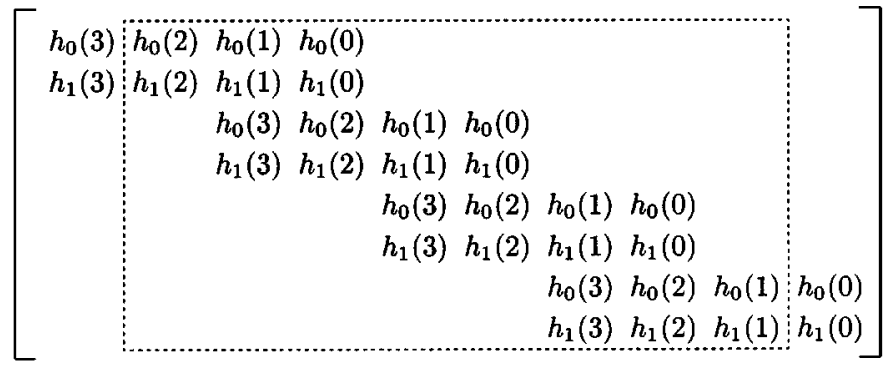

Fig. 2. Analysis matrix $\boldsymbol{F}$ and its truncation. $M=2, N=8$, length- 4 filters. The matrix is to be truncated to the part surrounded by the dashed box.

of $\tilde{\boldsymbol{H}}_{k}$ can be written as linear combinations of those of $\boldsymbol{H}_{k}$. With quadratic matrices $\boldsymbol{U}_{k}$, these linear combinations can be expressed as $\tilde{\boldsymbol{H}}_{k}=\boldsymbol{U}_{k} \boldsymbol{H}_{k}$ for the analysis and $\tilde{\boldsymbol{G}}_{k}=\boldsymbol{G}_{k} \boldsymbol{U}_{k}^{-1}$ for the synthesis side. The modified analysis and synthesis equations then become

$$
\begin{aligned}
\boldsymbol{v}_{k} & =\tilde{\boldsymbol{H}}_{k} \boldsymbol{x}=\boldsymbol{U}_{k} \boldsymbol{H}_{k} \boldsymbol{x}, \\
\hat{\boldsymbol{x}} & =\sum_{k=1}^{3} \tilde{\boldsymbol{G}}_{k} \boldsymbol{v}_{k}=\sum_{k=1}^{3} \boldsymbol{G}_{k} \boldsymbol{U}_{k}^{-1} \boldsymbol{v}_{k}
\end{aligned}
$$

with $U_{2}=\boldsymbol{I}$. Since we are interested in maintaining orthogonality during optimization, the matrices $\boldsymbol{U}_{k}$ must satisfy $\boldsymbol{U}_{k}^{-1}=\boldsymbol{U}_{k}^{T}$.

\section{Avoiding DC Leakage}

The boundary filters designed with the Gram-Schmidt procedure will usually suffer from dc leakage into all subbands, because all truncated impulse responses typically have nonzero mean and the designed filters are found as linear combinations of the truncated ones. In the following, we derive restrictions on the matrices $\boldsymbol{U}_{1}$ and $\boldsymbol{U}_{3}$ to avoid dc leakage. For the reason of simplicity, we only look at the left boundary and thus at $U_{1}$. We first generate a basis vector that represents a dc signal in the row space of $\boldsymbol{H}_{1}$. Then we use the Gram-Schmidt procedure to complete $\boldsymbol{H}_{1}$. The remaining optimization steps are carried out in such a way that we have control over the dc component of an input signal.

Let $\overline{\boldsymbol{H}}_{1}$ be a matrix that contains a basis for the row space of $\boldsymbol{H}_{1}$. Typically, $\overline{\boldsymbol{H}}_{1}$ can be generated by taking the upper part of the truncated matrix $\boldsymbol{F}$. It does not need to be an orthogonal matrix, but it must have maximum rank, so that its rows span the entire subspace of left boundary filters. Further, let $\boldsymbol{t}$ be a length- $N$ vector of ones: $\boldsymbol{t}=[1,1, \ldots, 1]^{T}$. We now compute the orthogonal projection of $\boldsymbol{t}$ onto the row space of $\overline{\boldsymbol{H}}_{1}$

$$
\hat{\boldsymbol{t}}:=\overline{\boldsymbol{H}}_{1}^{T}\left[\overline{\boldsymbol{H}}_{1} \overline{\boldsymbol{H}}_{1}^{T}\right]^{-1} \overline{\boldsymbol{H}}_{1} \boldsymbol{t} .
$$

The first row of the matrix $\boldsymbol{H}_{1}$ is then chosen as $\hat{\boldsymbol{t}}^{T}$. All further rows of $\boldsymbol{H}_{1}$ can be found via the Gram-Schmidt procedure, using the rows of $\overline{\boldsymbol{H}}_{1}$ as a given basis for the subspace in question. Note that one of the rows of $\overline{\boldsymbol{H}}_{1}$ will not be needed, because $\hat{\boldsymbol{t}}^{T}$ has been included, which already is a linear combination of the rows of $\overline{\boldsymbol{H}}_{1}$. The inverse in (9) always exists because $\overline{\boldsymbol{H}}_{1}$ and $\boldsymbol{H}_{1}$ have the same row space and $\boldsymbol{H}_{1}$ is orthogonal.

The matrix $\boldsymbol{H}_{1}$ constructed with the above algorithm has the property that all its rows, except the first one, have zero mean. This property is easily kept by choosing $U_{1}$ as

$$
\boldsymbol{U}_{1}=\left[\begin{array}{cc}
1 & 0 \\
0 & V_{1}
\end{array}\right]
$$

where $\boldsymbol{V}_{1}$ is orthogonal. More details on the choice of $\boldsymbol{V}_{1}$ will be given in Section III-B. The same concept can be used for the right boundary.

\section{BOUNDARY FILTER OPTIMIZATION}

\section{A. Unconstrained Maximization of the Coding Gain}

We interpret the subband decomposition according to (8) as a unitary transform that maps $N$ input values into $N$ transform coefficients. Under the assumption of a high bit rate and uncorrelated quantization errors, the coding gain may then be expressed as [23], [24]

$$
G=\sigma_{x}^{2} \prod_{\ell=0}^{N-1}\left(\sigma_{v_{\ell}}^{2}\right)^{-1 / N}
$$

where $\sigma_{v_{\ell}}^{2}$ are the variances of the subband samples computed via (8). Thus, optimizing the boundary filters to yield maximum coding gain turns out to be equivalent to minimizing the products of the diagonal elements of

$$
\boldsymbol{R}_{v_{k} v_{k}}=\boldsymbol{U}_{k} \boldsymbol{H}_{k} \boldsymbol{R}_{x x} \boldsymbol{H}_{k}^{T} \boldsymbol{U}_{k}^{T}, \quad k=1,3 .
$$

The matrices $\boldsymbol{R}_{v_{k}, v_{k}}$ are the autocorrelation matrices of the subband samples $\boldsymbol{v}_{k}$, generated from an input process $\boldsymbol{x}$ with autocorrelation matrix $\boldsymbol{R}_{x x}$. Minimizing the product of the diagonal elements is accomplished by the Karhunen-Loève transforms (KLTs) of the processes $\boldsymbol{y}_{k}$. In other words, the rows of the optimal matrices $\boldsymbol{U}_{k}, k=1,3$ are the transposed eigenvectors of

$$
\boldsymbol{R}_{y_{k} y_{k}}=\boldsymbol{H}_{k} \boldsymbol{R}_{x x} \boldsymbol{H}_{k}^{T} .
$$

The eigenvalue problem is easily solved and no numerical optimization is required to find the optimal boundary filters.

Note that the KLT not only maximizes the coding gain; it also minimizes the approximation error when reconstruction is carried out from a subset of the transform coefficients [23]. For this, the coefficients used for reconstruction have to be the ones that are computed with the eigenvectors that correspond to the largest eigenvalues.

\section{B. Maximizing the Coding Gain Under the Zero-Mean Constraint}

To obtain boundary filters with maximum coding gain under the zero-mean constrain, we use the parameterization (10). Again, the key to the solution is the KLT. We partition $H_{1}$ as

$$
\boldsymbol{H}_{1}=\left[\begin{array}{c}
\hat{\boldsymbol{t}}^{T} \\
\hat{\boldsymbol{H}}_{1}
\end{array}\right]
$$

and, following the same ideas as in Section III-A, we find the rows of the optimal matrix $\boldsymbol{V}_{1}$ to be the transposed eigenvectors of the matrix

$$
\hat{\boldsymbol{R}}_{y_{k} y_{k}}=\hat{\boldsymbol{H}}_{k} \boldsymbol{R}_{x x} \hat{\boldsymbol{H}}_{k}^{T} .
$$

\section{Approximating the Frequency Responses of the Original Filters}

The previous two subsections have shown how the coding gain can be maximized through the choice of boundary filters. The described design methods, although optimal, usually do not lead to boundary filters that have similar frequency responses as the original filters. Assuming a total number of $L_{k}$ boundary filters and an AR(1) input process, we may expect the optimal boundary filters to be bandpass filters with $L_{k}$ different passbands in the frequency range $[0, \pi]$. This is justified by the fact that the direct application of the KLT to such a process results in a similar behavior. Thus, the frequency range $[0, \pi]$ will be divided into $L_{k}$ bands at the boundaries, instead of having $M$ bands like the original filters. To convert the boundary filters into new ones with similar time-frequency resolutions as the original filters, we take linear combinations of the previously constructed boundary filters. In the following, we give a straightforward description of the method. The rationale behind it is outlined in the Appendix.

Let $L_{k}=\nu_{k} M$ with $\nu_{k} \in \mathbb{Z}$, and let $\hat{\boldsymbol{h}}_{i, k}^{T}, i=1,2, \ldots, L-1$ denote the $i$ th row of $\tilde{\boldsymbol{H}}_{k}=\boldsymbol{U}_{k} \boldsymbol{H}_{k}$. Let us assume that the rows of $\boldsymbol{U}_{k}$ 


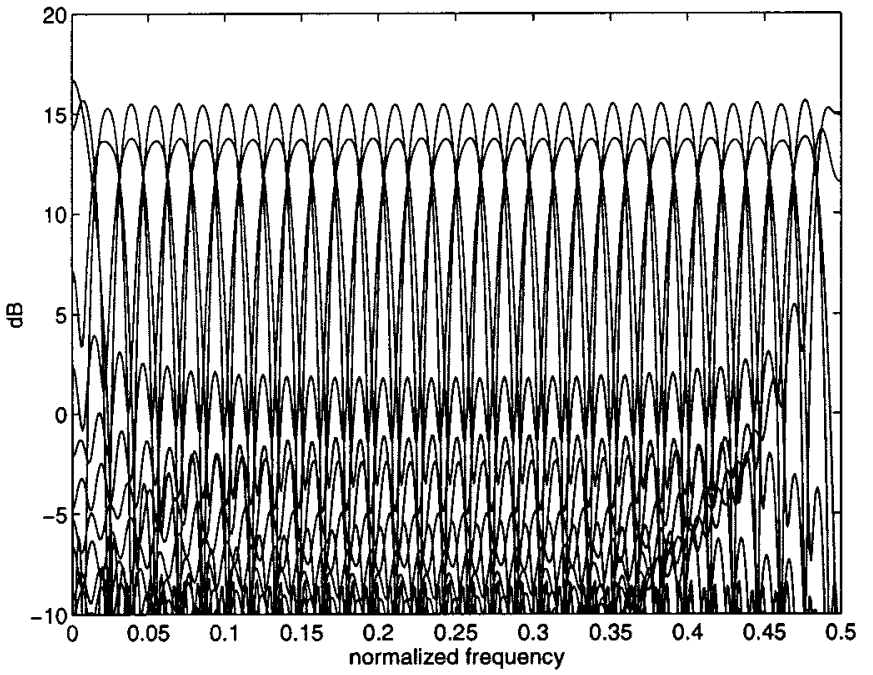

(a)

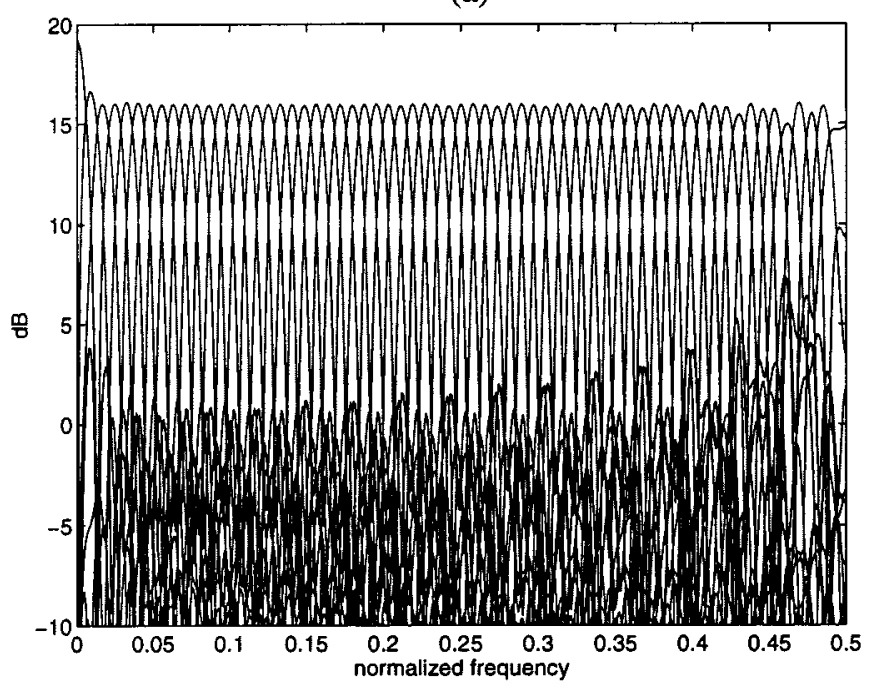

(c)

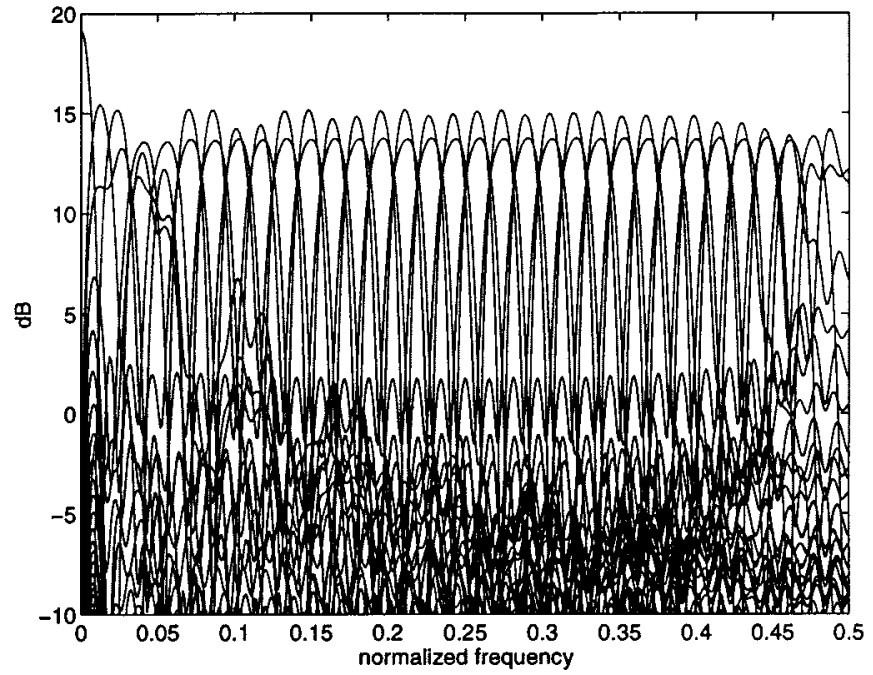

(b)

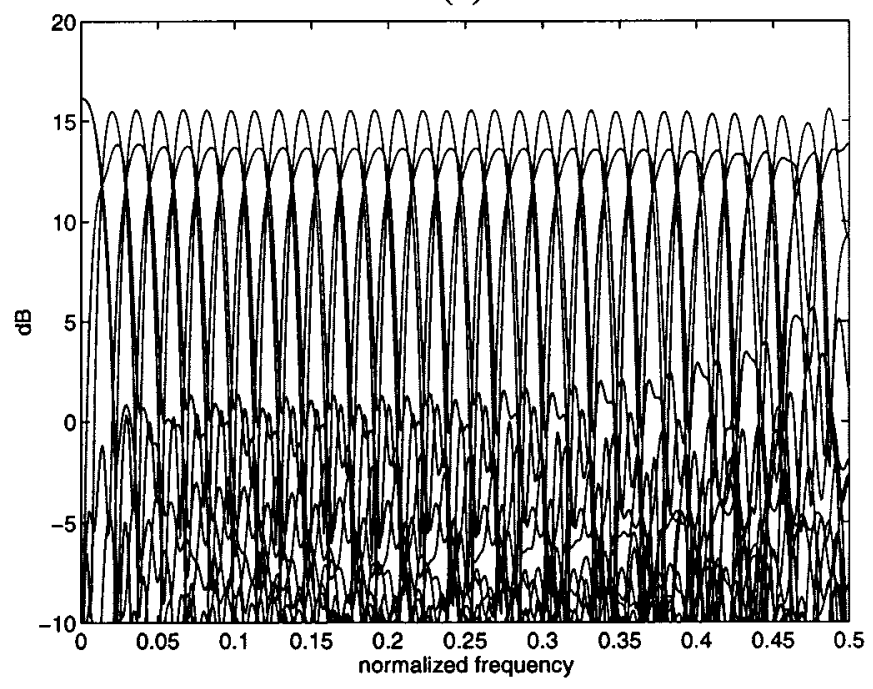

(d)

Fig. 3. Frequency responses of left boundary filters. (a) Gram-Schmidt method, (b) Gram-Schmidt method and zero-mean constraint, (c) maximum coding gain under zero-mean constraint, and (d) boundary filters that resemble the frequency responses of the original ELT filters. Note: the significant sidelobes of some filters in (c) and (d) near the normalized frequency 0.5 belong to high-indexed subbands, not to subbands near the low-pass one.

had been ordered according to the corresponding eigenvalues of $\boldsymbol{R}_{y_{k}, y_{k}}$ or $\hat{\boldsymbol{R}}_{y_{k}, y_{k}}$, depending on the method used. We assume that the first row corresponds to the largest and the last one to the smallest eigenvalue. Let $\boldsymbol{A}_{k}$ be orthogonal matrices of size $\nu_{k} \times \nu_{k}$. The new filters are constructed as

$$
\left[\begin{array}{c}
\tilde{\boldsymbol{h}}_{(i-1) \nu_{k}+1, k}^{T} \\
\vdots \\
\tilde{\boldsymbol{h}}_{i \nu_{k}, k}^{T}
\end{array}\right]=\boldsymbol{A}_{k}\left[\begin{array}{c}
\hat{\boldsymbol{h}}_{(i-1) \nu_{k}+1, k}^{T} \\
\vdots \\
\hat{\boldsymbol{h}}_{i \nu_{k}, k}^{T}
\end{array}\right], \quad i=1,2, \ldots, M
$$

where $\tilde{\boldsymbol{h}}_{i, k}^{T}$ forms the $i$ th row of the final optimized analysis matrix $\tilde{\tilde{\boldsymbol{H}}}_{k}$.

The orthogonal matrices $\boldsymbol{A}_{k}$ should be chosen in such a way that their columns, when interpreted as filter impulse responses, are bandpass filters that divide the frequency range $[0, \pi]$ into $\nu_{k}$ bands of equal widths. For $\nu_{k}=2$, the matrix $\boldsymbol{A}_{k}$ would typically be

$$
\boldsymbol{A}_{k}=\frac{1}{\sqrt{2}}\left[\begin{array}{rr}
1 & 1 \\
1 & -1
\end{array}\right]
$$

For $\nu_{k}>2$, the $\nu_{k} \times \nu_{k}$ discrete cosine transform (DCT)-II matrices are possible choices. Completely parameterizing $\boldsymbol{A}_{k}$ as orthogonal matrices and optimizing the free parameters according to some criteria, however, may lead to an improvement over the DCT-II matrices.

Consider the case where the boundary filters are constructed in such a way that the dc component of the input signal is concentrated in a single subband coefficient: $\boldsymbol{U}_{k} \boldsymbol{H}_{k} \boldsymbol{t}=[\gamma, 0, \ldots, 0]$ with some value $\gamma$. If $\boldsymbol{A}_{k}$ is now chosen such that its first column contains a constant, the filters $\hat{\boldsymbol{h}}_{i, k}, i=1, \ldots, \nu_{k}$ will have equal mean values. All other filters for $i>\nu_{k}$ will have zero mean.

Note that the operation (15) is supposed to turn the narrow-band filters $\hat{\boldsymbol{h}}_{i, k}$ into wide-band ones $\tilde{\boldsymbol{h}}_{i, k}$ with different time localizations. The new filters are not necessarily ordered according to their time localizations. If such an ordering is wanted, it can be carried out in an additional step.

\section{DESIGN EXAMPLES}

We consider a paraunitary cosine-modulated 32-band filter bank with extended lapped transform (ELT) prototype according to [25]. In this filter bank, the subband filters have nonlinear phase, so that 


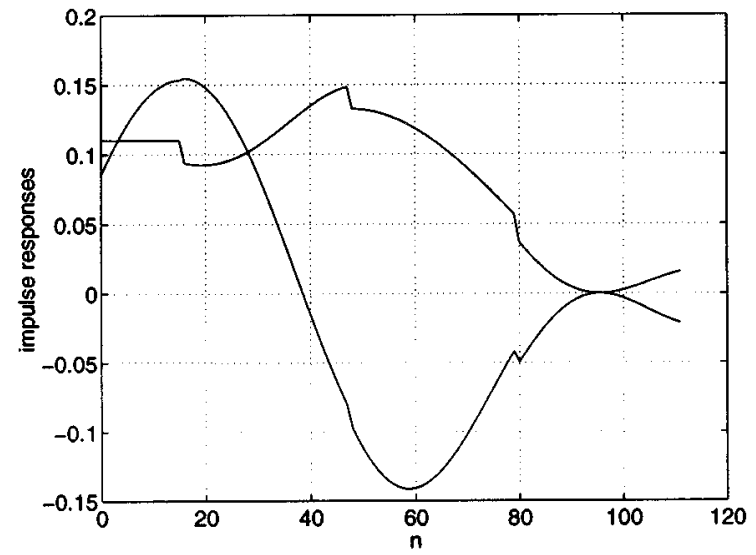

(a)

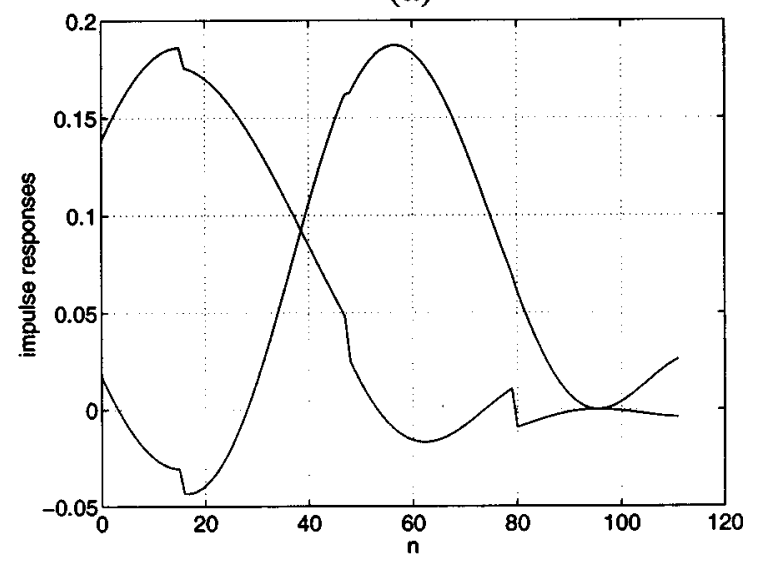

(b)

Fig. 4. Impulse responses of right boundary filters that resemble the frequency responses of the original filters. (a) Filters in $\hat{\boldsymbol{h}}_{1,1}$ and $\hat{\boldsymbol{h}}_{2,1}$. (b) Filters in $\tilde{\boldsymbol{h}}_{1,1}$ and $\tilde{\boldsymbol{h}}_{2,1}$.

symmetric reflection techniques cannot be applied and boundary filters must be used. ELT filters have filter length $4 M$, and the total number of boundary filters for the left-hand side turns out to be $L=2 M$. On the right-hand side, their number depends on the parameter $s$ used to describe $N$ in (1).

We first look at the left boundary. A first set of boundary filters was designed via the Gram-Schmidt procedure. The frequency responses of the boundary filters are depicted in Fig. 3(a). As the plot shows, in this example, the Gram-Schmidt procedure directly yields boundary filters with relatively good frequency selectivity. The filters divide the frequency range $[0, \pi]$ into $M$ bands, and there are always two filters with the same passband, but different time localizations. A weakness of the method is that several boundary filters, in addition to the two low-pass ones, have large nonzero mean. Assuming an AR(1) input process with correlation coefficient $\rho=0.9$, the coding gain of these filters is 6.919 $\mathrm{dB}$. A second set of boundary filters was designed under the zero-mean constraint, according to the method in Section II-C. Their frequency responses are depicted in Fig. 3(b). It can be seen that many of these filters have poor frequency selectivity, and the coding gain is only $6.52 \mathrm{~dB}$. A third set of boundary filters was designed to maximize the coding gain for the above mentioned AR(1) process under the zero-mean constraint. The frequency responses of the filters are shown in Fig. 3(c). It can be seen that the $2 M$ boundary filters have $2 M$ disjoint passbands with good frequency selectivity. The coding gain is $7.337 \mathrm{~dB}$. For comparison, the gain that can be obtained through an unconstrained optimization with the method in Section III-A amounts to 7.341 dB. Finally,

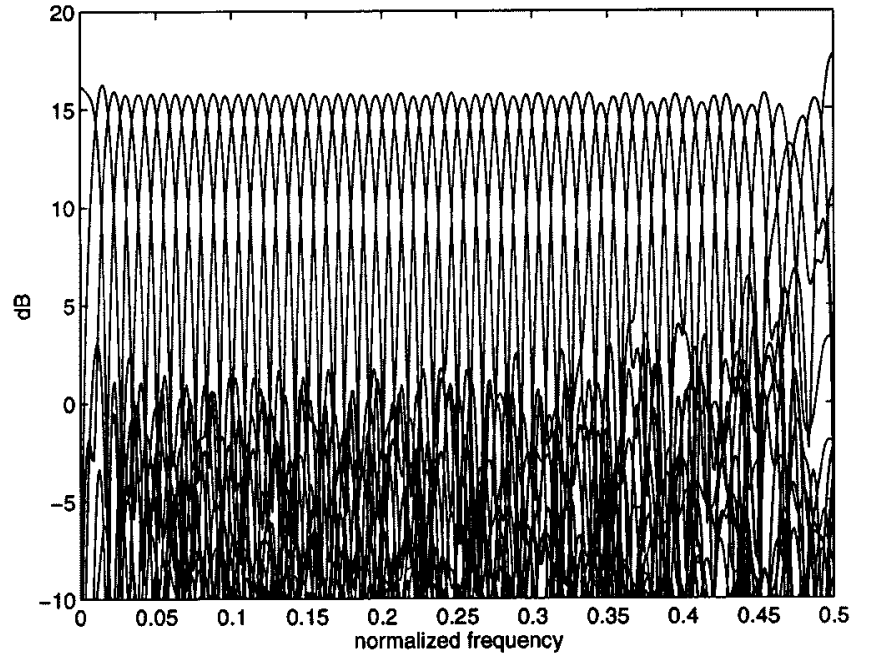

Fig. 5. Frequency responses of boundary analysis filters with maximum coding gain under the zero-mean constraint, designed for a signal length $N=K M+s$ with $M=32$ and $s=28$.

the designed filters were converted into filters with only $M$ passbands, using the algorithm described in Section III-C with $\boldsymbol{A}_{k}$ as in (16). The frequency responses are depicted in Fig. 3(d). These filters no longer maximize the coding gain, but they have similar frequency responses as the original filters, allowing for the use of the same bit allocation in the center and at the boundaries of a signal. In this example, the coding gain amounts to $7.327 \mathrm{~dB}$, which means that the reduction in coding gain due to this manipulation is only marginal. To demonstrate the effects in the time domain, Fig. 4 depicts the impulse responses contained in $\hat{\boldsymbol{h}}_{1,1}, \hat{\boldsymbol{h}}_{2,1}$, and $\tilde{\boldsymbol{h}}_{1,1}, \tilde{\boldsymbol{h}}_{2,1}$, respectively.

We now consider the processing of the right boundary. Boundary filters were designed for various signal lengths. It turned out that frequency-selective filters were obtained for all $N$ and not only for $N=$ $K M$. To give an example, Fig. 5 shows the frequency responses of boundary filters with maximum coding gain for $M=32$ and $N=$ $K M+28$, obtained under the zero-mean constraint.

\section{CONCLUSIONS}

The methods presented in this paper enable the design of orthogonal, perfect reconstruction boundary filters with ideal dc behavior and maximum coding gain. The signal lengths can be chosen independent of the number of channel of the filter bank. This allows for segmented coding where the segmentation can take place at arbitrary points. For example, by segmenting audio signals direct in front of attacks, the problem of pre-echoes can be avoided. This paper has also proposed a method to find boundary filters that have similar frequency responses as the original filters. This allows for the use of the same bit allocation in the center and at the boundaries of a signal. All methods presented provide direct solutions and need no cost-intensive numerical optimization.

\section{APPENDIX}

In the following, we outline the rationale behind the boundary filter manipulation described in Section III-C. Consider the construction of filters $p_{\ell}(n)$ as linear combinations of time-shifted versions of a given filter $h(n)$

$$
p_{\ell}(n):=\sum_{k=0}^{\nu-1} \alpha_{k, \ell} h(n-k M), \quad \ell=0, \ldots, \nu-1
$$


where $\alpha_{k, \ell}$ are arbitrary weights. The frequency responses of the filters $p_{\ell}(n)$ are

$$
P_{\ell}\left(e^{j \omega}\right)=H\left(e^{j \omega}\right) A_{\ell}\left(e^{j \omega M}\right)
$$

with

$$
A_{\ell}\left(e^{j \omega}\right)=\sum_{k=0}^{\nu-1} \alpha_{k, \ell} e^{-j \omega k} .
$$

If $H\left(e^{j \omega}\right)$ has bandwidth $\pi / M$ and the filters $A_{\ell}\left(e^{j \omega}\right)$ have bandwidths $\pi / \nu$, then the filters $P_{\ell}\left(e^{j \omega}\right)$ will have bandwidths $\pi /(\nu M)$. The operation (17), which describes the construction of narrow-band filters $p_{\ell}(n)$ from time-shifted versions of a wide-band filter $h(n)$, can be written as

$$
\boldsymbol{P}=\boldsymbol{A}^{T} \boldsymbol{H}
$$

The rows of $\boldsymbol{P}$ and $\boldsymbol{H}$ contain the impulse responses $p_{\ell}(n)$ and $h(n-$ $k M$ ), respectively, and $\boldsymbol{A}$ contains the coefficients $\alpha_{k, \ell}$. Assuming an orthogonal matrix $\boldsymbol{A}$, the opposite is achieved with the operation

$$
\boldsymbol{H}=\boldsymbol{A P} .
$$

Equation (20) describes the operation in (15), which is used to turn narrow-band filters into wide-band ones with different time localizations.

\section{REFERENCES}

[1] J. Princen and J. D. Johnston, "Audio coding with signal adaptive filterbanks," in Proc. IEEE Int. Conf. Acoust., Speech, Signal Processing, Detroit, MI, 1995, pp. 3071-3074.

[2] D. Sinha and J. D. Johnston, "Audio compression at low bit rates using a signal adaptive switched filterbank," in Proc. IEEE Int. Conf. Acoust., Speech, Signal Processing, vol. 2, Atlanta, GA, 1996, pp. 1053-1056.

[3] J. Kliewer and A. Mertins, "Audio subband coding with improved representation of transient signal segments," in Proc. Eur. Signal Processing Conf., Rhodos, Greece, Sept. 1998, pp. 2345-2348.

[4] J. Kliewer, T. Karp, and A. Mertins, "Processing arbitrary-length signals with linear-phase cosine-modulated filter banks," Signal Process., vol. 80, pp. 1515-1533, Aug. 2000.

[5] K. N. Ngan, T. Meier, and D. Chai, Advanced Video Coding: Principles and Techniques, Advances in Image Communication, 7. Amsterdam, the Netherlands: Elsevier Science, 1999.

[6] MPEG-4 Video Verification Model, Version 14. Generic Coding of Moving Pictures and Associated Audio, ISO/IEC JTC1/SC 29/WG 11, 1999.

[7] J. Woods and S. O'Neil, "Subband coding of images," IEEE Trans. Acoust., Speech, Signal Processing, vol. ASSP-34, pp. 1278-1288, May 1986.

[8] M. J. T. Smith and S. L. Eddins, "Analysis/synthesis techniques for subband coding," IEEE Trans. Acoust., Speech, Signal Processing, pp. 1446-1456, Aug. 1990.

[9] J. N. Bradley, C. M. Brislawn, and V. Faber, "Reflected boundary conditions for multirate filter banks," in Proc. Int. Symp. Time-Frequency and Time-Scale Analysis, Canada, 1992, pp. 307-310.

[10] H. J. Barnard, J. H. Weber, and J. Biemond, "Efficient signal extension for subband/wavelet decomposition of arbitrary length signals," in Proc. SPIE VCIP, vol. 2094, Nov. 1993, pp. 966-975.

[11] L. Chen, T. Q. Nguyen, and K. P. Chan, "Symmetric extension methods for $M$-channel linear-phase perfect reconstruction filter banks," IEEE Trans. Signal Processing, vol. 43, pp. 2505-2511, Nov. 1995.

[12] R. L. de Queiroz, "Subband processing of finite length signals without border distortions," in Proc. IEEE Int. Conf. Acoust., Speech, Signal Processing, vol. IV, San Francisco, CA, Mar. 1992, pp. 613-616.

[13] C. Herley, J. Kovačević, K. Ramchandran, and M. Vetterli, "Tilings of the time-frequency plane: Construction of arbitrary orthogonal bases and fast tiling algorithms," IEEE Trans. Signal Processing, vol. 41, pp. 3341-3359, Dec. 1993

[14] C. Herley, "Boundary filters for finite-length signals and time-varying filter banks," IEEE Trans. Circuits Syst. II, vol. 42, pp. 102-114, Feb. 1995.
[15] A. Mertins, "Time-varying and support preservative filter banks: Design of optimal transition and boundary filters via SVD," in Proc. IEEE Int. Conf. Acoust., Speech, Signal Processing, Detroit, MI, May 1995, pp. 1316-1319.

[16] T. Kalker, "On optimal boundary and transition filters in time-varying filter banks," in Proc. IEEE Int. Conf. Image Processing, vol. 1, 1996, pp. 625-628.

[17] R. L. de Queiroz and K. R. Rao, "Optimal orthogonal boundary filter banks," in Proc. IEEE Int. Conf. Acoust., Speech, Signal Processing, vol. II, Detroit, MI, May 1995, pp. 1296-1299.

[18] M. Coffey, "Boundary compensated wavelet bases," in Proc. IEEE Int. Conf. Acoust., Speech, Signal Processing, München, Germany, May 1997, pp. 2129-2132.

[19] A. Mertins, "Optimized biorthogonal shape adaptive wavelets," in Proc. IEEE Int. Conf. Image Processing, vol. 3, Chicago, IL, Oct. 1998, pp. 673-677.

[20] W. Niehsen, "Boundary filters without DC leakage for paraunitary filter banks," in Proc. IEEE Int. Conf. Acoust., Speech, Signal Processing, vol. 3, Phoenix, AZ, Mar. 1999, pp. 1465-1468.

[21] A. Mertins, "Boundary filter optimization for segmentation-based subband coding," IEEE Trans. Signal Processing, 2001, to be published.

[22] V. Nuri and R. H. Bamberger, "Size limited filter banks for subband image compression," IEEE Trans. Image Processing, vol. 4, pp. 1317-1323, Sept. 1995.

[23] N. S. Jayant and P. Noll, Digital Coding of Waveforms. Englewood Cliffs, NJ: Prentice-Hall, 1984.

[24] J. Katto and Y. Yasuda, "Performance evaluation of subband coding and optimization of its filter coefficients," in Proc. SPIE Visual Communication and Image Processing, Nov. 1991, pp. 95-106.

[25] H. S. Malvar, "Extended lapped transforms: Fast algorithms and applications," IEEE Trans. Signal Processing, vol. 40, pp. 2703-2714, Nov. 1992.

\section{Design of Adaptive Envelope-Constrained Filters in the Presence of Impulsive Noise}

Wei Xing Zheng

\begin{abstract}
The problem of designing adaptive filters subject to output envelope constraints in the presence of impulsive noise at the input channel is investigated. Median smoothing is incorporated into the recently developed adaptive envelope-constrained filtering algorithms with a view to suppressing the effect of impulsive noise. It is demonstrated that the proposed adaptive filtering algorithms can provide notable improvements in the performance. In particular, the output envelope constraints of primary interest can be more easily satisfied by the filter weight estimate obtained with the median smoothing than with the average smoothing in impulsive noise situations. Numerical simulations are included to show the effectiveness of the proposed adaptive filtering algorithms.
\end{abstract}

Index Terms-Adaptive filtering, channel equalizers, envelope-constrained filters, impulsive noise.

\section{INTRODUCTION}

Envelope-constrained (EC) filters define a class of linear finite impulse response (FIR) filters whose output in response to a given input pulse will yield the desired pulse shape to within the preset design tolerance.

Manuscript received December 1999; revised January 2001. This work was supported in part by the Australian Research Council under a Research Grant and in part by the University of Western Sydney, Nepean, Australia, under a Research Grant. This paper was recommended by Associate Editor A. Skodras.

The author is with the School of Quantitative Methods and Mathematical Sciences, University of Western Sydney, Kingswood, NSW 2747, Australia (e-mail: w.zheng@uws.edu.au).

Publisher Item Identifier S 1057-7130(01)03053-1. 